\title{
Investigation of Disease-causing Mutations in Sucrase-Isomaltase
}

\author{
A Jewczynko ${ }^{1}$, D Rose ${ }^{2}, \mathrm{~N} \mathrm{Nava}^{3}$ \\ ${ }^{1}$ University of Waterloo, Toronto, ON, ${ }^{2}$ Dept of Biology, Univ of Waterloo, ${ }^{3}$ University of Waterloo, \\ Waterloo \\ aejewczy@uwaterloo.ca
}

Sucrase-Isomaltase (SI) is an intestine-localized eukaryotic enzyme that breaks down a variety of disaccharides and oligosaccharides into monosaccharides, through the cleavage of $\alpha-1,2, \alpha-1,4$ and $\alpha-1,6$ glycosidic bonds. Congenital Sucrase-Isomaltase Deficiency (CSID) is a genetic metabolic disorder which affects the expression or function of SI and impacts an individual's ability to digest certain carbohydrates. Symptoms of CSID include diarrhea, abdominal pain and an intolerance to carbohydrates such as sucrose and starch. Common CSID mutations found in 83\% of CSID patients of European descent include G1073D, V577G, F1745C and R1124X.

The objective of this project is to investigate the disease-causing mutations of SI. The effect of a single change of residue may affect the stability of SI and/or a structural feature which is responsible for its enzymatic activity. The CSID protein mutants will be investigated by testing the protein stability and substrate affinity to determine the cause of the lack of enzymatic activity. The stability of the expressed CSID protein mutants will be assessed through exposure to a variety of temperature and $\mathrm{pH}$, over different periods of time to determine whether the lack of enzymatic activity is a result of a lack of structural integrity. Since these point mutations are not located within the catalytic active site, measuring the substrate affinity will help clarify whether the lack of enzymatic activity is a result of an indirect structural effect on the catalytic site or a lack of substantial substrate affinity. These investigations into protein stability and substrate affinity of the protein mutants will provide a clearer insight on the effect of these disease-causing mutations on the structure and function of SI.

Acta Cryst. (2020). A76, a180 